\title{
Optical switching in nano-arrays: Transistor action through directed energy transfer
}

\author{
Richard G. Crisp and David L. Andrews* \\ Nanostructures and Photomolecular Systems, School of Chemical Sciences and Pharmacy, \\ University of East Anglia, Norwich NR4 7TJ, UK
}

\begin{abstract}
Resonance energy transfer (RET) is a near-field mechanism for propagating optical energy between particles with suitably matching frequency response. The process communicates electronic excitation between suitably disposed (donor and acceptor) dipoles in close proximity, activated on excitation of the donor. In a multi-component system the transfer of excitation between any given donor and acceptor is usually passive, and it competes with loss mechanisms such as radiative decay and the possibility of transfer to one or more other acceptors. It thus appears that any potential exploitation of RET for optical switching is compromised by the innate passivity of the process. Now it emerges that there is a direct, all-optical route to introduce the necessary control. In a system constructed to satisfy frequencymatching conditions, but designedly to inhibit RET by geometric configuration, the throughput of laser pulses can facilitate energy transfer processes that would otherwise be forbidden, by laser-assisted resonant energy transfer. Suitably configuring an arrangement of transition dipoles, it proves possible to design parallel planar arrays of optical donor and acceptor particles such that the transfer of energy from any single donor, to its counterpart in the opposing plane, can be switched by appropriate laser radiation. As the energy transfer is itself mediated electromagnetically, the device operates as an optical transistor. For simplicity, a pair of two-dimensional arrays is envisaged, each consisting of equally spaced, identical particles arranged on a square lattice. A detailed appraisal of the system, including a consideration of competing processes, suggests that this configuration offers a new basis for the design of optically activated nanoscale transistor arrays.
\end{abstract}

Keywords: Molecular nanostructures, optoelectronic device, optical computing, optical transistor, resonant energy transfer, non-linear optics

\section{INTRODUCTION}

In the drive to miniaturize ultrafast optical switching and interconnect devices, there is much current interest in nanoscale designs where atomic or molecular assemblies can operate with a similar functionality to established microdevices. ${ }^{1-5}$ One possible basis for such a design is to exploit the transient coupling of throughput optical radiation. In particular, attention focuses on the near-field mechanism for propagating optical energy between particles with suitably matching frequency response, via the process of resonant energy transfer (RET). This process communicates electronic excitation between suitably disposed (nominally donor and acceptor) dipoles in close proximity, activated on excitation of the donor. However, in a multi-component system the transfer of excitation between any given donor and acceptor is usually passive, and it competes with loss mechanisms such as radiative decay and the possibility of transfer to an undesignated acceptor. The exploitation of RET for optical switching thus appears to be inherently compromised by its innate passivity - a lack of suitable control mechanisms. Until recently it appeared that only by inefficient and kinetically limiting means, such as reorientation or movement of the coupled units, could significant control be effected. However, it now emerges that there is a direct and all-optical route to introduce the necessary control in RET, overcoming such obstacles. The mechanism, and the means of its implementation, is the subject of this paper.

In a system constructed to satisfy frequency-matching conditions, but designedly to inhibit RET by geometric configuration, it has been shown that the throughput of laser pulses can facilitate energy transfer that would otherwise be forbidden, through a process of laser-assisted resonant energy transfer (LARET). ${ }^{6}$ By suitably configuring an arrangement of transition dipoles, it proves possible to design parallel planar arrays of optical donor and acceptor particles such that the transfer of energy from any single donor, to its counterpart in the opposing plane, can be switched 
by throughput laser radiation of an appropriate intensity, frequency and polarization. As the resonance energy transfer is itself mediated electromagnetically, the device operates as an optical transistor. For simplicity, a pair of twodimensional arrays is envisaged, each consisting of equally spaced, identical particles arranged on a square lattice. A detailed appraisal of the system reveals an intricate interplay of electronic structure, optical frequency and geometric factors. The switching radiation must be significantly off-resonant with regard to the donors and acceptors (to disallow direct coupling of the transitions between the excited and ground states of the particles), and the latter units must each have an electronic response that needs at least a three-level model for its accurate representation. Quantitative assessments reveal that, under expeditious conditions and with a relatively modest laser intensity of $10^{14} \mathrm{~W} \mathrm{~m}^{-2}$, transfer from a given donor in the presence of laser light delivers excitation almost exclusively to its counterpart acceptor typically $10^{5}$ times more effectively than for transfer to any other acceptor in the array. Although the transfer efficiency in such a system is high, a number of competing processes represent possible sources of information loss. Adopting a principle widely used in multichromophore systems for conventional RET, back-transfer from acceptor to donor can be precluded by engineering differences in the absorption and emission profiles of the particles. The sought advantage of unidirectionality that is ensured by this spectroscopic gradient is offset by a reduction in efficiency necessarily associated with small losses of energy, possibly necessitating the adoption of appropriate cooling measures in device applications. The other intrinsic complication, a finite probability for energy transfer from the designated acceptor to another acceptor, can be addressed by configuration of the relative spacing of the donor and acceptor arrays, relative to the internal spacing of the particles in each array. It is submitted that the results promise a new basis for the design of optically activated transistor action in nanoscale components.

In the following, the principles and key equations for RET and LARET are first established in Section 2. The results are developed for application to a pair of parallel arrays in Section 3, and in Section 4 the significance of the effect is quantitatively assessed. The concluding discussion realistically appraises possible device applications.

\section{PRINCIPLES}

In this Section, the key principles, definitions and parameters for RET and LARET are introduced, and the relevant equations are presented. Detail of the underlying development of theory based on quantum electrodynamics (QED) are to be found in earlier, cited publications by the QED group at the University of East Anglia.

\subsection{RET}

The pairwise transfer of energy between two chromophores $D$ and $A$ can be described by the chemical equation:

$$
D^{*}+A \longrightarrow D+A^{*},
$$

where $\xi$ denotes an excited state for chromophore $\xi$. Chromophore $D$ is designated the donor and $A$ the acceptor. For the description of this event, neither the mechanism for the excitation of $D$ nor the subsequent decay of $A$ comes into play; those are kinetically separable events. Since the energy transfer takes place between chromophores which are closely spaced (but beyond significant wavefunction overlap), it is generally mediated by a coupling of the transition dipole moments for the donor decay and acceptor excitation. Using the Fermi Rule, the short-range limit of the associated probability at time $t$ is given by, ${ }^{7,8}$

$$
P=\left(\frac{\left|\mu_{A}^{0 \alpha}\right|^{2}\left|\mu_{B}^{\beta 0}\right|^{2} \rho_{f} t}{8 \pi \hbar \varepsilon_{0}^{2}}\right) \frac{\kappa^{2}}{R^{6}},
$$

where $R$ is the separation of the chromophores, $\mu_{\xi}^{f i}$ the transition-dipole moment of chromophore $\xi, \alpha$ and $\beta$ label the donor and acceptor excited states, and $\rho_{\mathrm{f}}$ is the density of final states. Also, $\kappa$ is an orientation factor defined as $\cos \psi-3 \cos \theta \cos \phi$, in which $\psi$ is the angle between the two transition moments and $\theta, \phi$ are the angles of $\boldsymbol{\mu}_{A}^{0 \alpha}$ and $\boldsymbol{\mu}_{B}^{\beta 0}$ respectively with $\boldsymbol{R}$, the displacement vector of magnitude $R$. For a given pair of chromophores with suitably 
matching energy profiles, the transfer efficiency is governed by both the separation of the pair and their relative orientation. The $\kappa^{2}$ factor ranges between 0 and 4 - effectively determining if energy transfer is geometrically allowed. This will be key to the optical switching effect described below - the facility to preclude RET, but permit LARET by a judicious choice of geometric configuration. It is in the $R^{-6}$ separation dependence that the other major influence over the transfer is observed, provided both transitions are allowed; even a small decrease in $R$ will greatly increase the probability of energy transfer. These considerations are fundamental to the work which follows.

The above analysis holds true for chromophores with perfectly overlapping energy levels. If the chromophores are chemically inequivalent, however, they are unlikely to have identical energy profiles and the spread of vibrational energy levels about each electronic level must be considered. Correspondingly, the probability expressed in eqn (2) can be written as follows, where $\hbar \omega$ is the energy transferred; ${ }^{9}$

$$
P=\frac{9 c^{4} \kappa^{2} t}{8 \pi \tau_{D} R^{6}} \int F_{D}(\omega) \sigma_{A}(\omega) \omega^{-4} \mathrm{~d} \omega
$$

where $\tau_{D}$ is the donor radiative lifetime, $\sigma_{A}(\omega)$ is the absorption cross-section of the acceptor, $F_{D}(\omega)$ the donor emission spectrum (here normalized to unity) and the integration is performed over a frequency range that is specifically limited to the salient donor emission and acceptor absorption regions.

\subsection{LARET}

Pairwise energy transfer can be effected by the application of intense radiation, in a process known as laser-assisted resonance energy transfer. ${ }^{6}$ The mechanism is one of cooperative forward Rayleigh scattering by the chromophore pair. In early work on LARET, the emphasis was on modifying the rate of RET in a configuration where both processes were allowed. Here the context is quite different: the introduction of a new channel permitting energy transfer only in the presence of the throughput radiation. However, it is still necessary to include the effect of RET in the analysis, in order to secure results of a general form that will be applicable not only to a chromophore pair whose orientation precludes RET, but also to other potential donor-acceptor pairings that fail to satisfy this special condition. Restricting consideration to chromophores which are non-polar, (for polar species another mechanism can operate: see ref. 6 for details), still two interaction pathways are possible - the laser radiation is absorbed at $D$ and emitted at $A$, or vice versa. In addition to the probability of RET as given by eqn (2), two additional terms arise. The first is the probability of LARET;

$$
P=\frac{\rho_{f} \int_{0}^{t} I^{2}(t) \mathrm{d} t}{32 \hbar c^{2} \pi \varepsilon_{0}^{4} R^{6}}\left|a^{\prime}+a^{\prime \prime}\right|^{2},
$$

where $I(t)$ is the irradiance of the laser beam and;

$$
\begin{aligned}
& a^{\prime}=\left\{e_{i} \alpha_{i j}^{D}\left(-k^{\prime}\right)\left(\delta_{j k}-3 \hat{R}_{j} \hat{R}_{k}\right) \alpha_{l k}^{A}\left(k^{\prime}\right) \bar{e}_{l}\right\}, \\
& a^{\prime \prime}=\left\{\bar{e}_{i} \alpha_{i j}^{D}\left(k^{\prime}\right)\left(\delta_{j k}-3 \hat{R}_{j} \hat{R}_{k}\right) \alpha_{l k}^{A}\left(-k^{\prime}\right) e_{l}\right\} .
\end{aligned}
$$

Here, $\boldsymbol{e}$ is the polarization vector of the laser radiation ( $\overline{\boldsymbol{e}}$ its complex conjugate) and $\alpha_{i j}^{\xi}$ is the (generalized) polarizability of chromophore $\xi$, expressible as;

$$
\alpha_{i j}^{f i(\xi)}\left( \pm k^{\prime}\right)=\sum_{r}\left[\frac{\mu_{i}^{f r} \mu_{j}^{r i}}{E_{f r} \pm \hbar c k^{\prime}}+\frac{\mu_{j}^{f r} \mu_{i}^{r i}}{E_{i r} \mp \hbar c k^{\prime}}\right] \text {. }
$$


Each of the two eqns (5) signifies one of the two interaction pathways for LARET. Finally, in configurations where both RET and LARET can operate, there is a probability arising from the quantum interference of the corresponding pathways, which can be written;

$$
P=\frac{\left|\mu_{A}^{0 \alpha}\right|\left|\mu_{B}^{\beta 0}\right| \kappa \rho_{f} \int_{0}^{t} I(t) \mathrm{d} t}{8 \hbar c \pi \varepsilon_{0}^{3} R^{6}} \mathfrak{R e}\left\{a^{\prime}+a^{\prime \prime}\right\}
$$

Previous investigations into this mechanism have revealed that, if geometrically allowed, the standard RET pathway is the dominant mechanism for energy transfer at irradiance levels much below $10^{16} \mathrm{~W} \mathrm{~m}^{-2}$. It is also important that the laser radiation is off-resonant (i.e. $\omega^{\prime} \neq \omega$ ), in order to prevent direct excitation of the acceptor.

\section{ARRAY STRUCTURE}

For technical applications it can be envisaged that a substantial number of individually addressable donor-acceptor pairs will form part of a larger integrated device. The crux of the design strategy is to optimally configure the donors and acceptors so as to satisfy two potentially conflicting conditions: (i) to arrange for each donor to have a counterpart acceptor to which excitation transfer is permitted only when throughput laser radiation is present; (ii) to ensure that for each donor, the probability of excitation transfer to any other non-designated acceptor is negligible. One solution that has been the subject of detailed theoretical analysis is a pair of parallel two-dimensional arrays. ${ }^{10}$ For simplicity, each array is considered to comprise equally spaced, identical particles arranged on a square lattice. Let $l$ be the distance between nearest neighbors in each array. The system is ascribed mutually orthogonal axes with unit vectors $\hat{i}, \hat{j}$ and $\hat{\boldsymbol{k}}$, such that $\hat{\boldsymbol{k}}$ defines the normal to both arrays, with the origin located on one of the acceptors. Each particle in the acceptor array is labelled $(u, v)$, where $u$ and $v$ are integers, and the corresponding displacement from the origin is $u \hat{i}+v \hat{j}$. The donor array is positioned such that every donor particle is directly above a corresponding particle in the acceptor array; the separation of the arrays is defined as $r$. A diagram of this system is given in figure 1 .

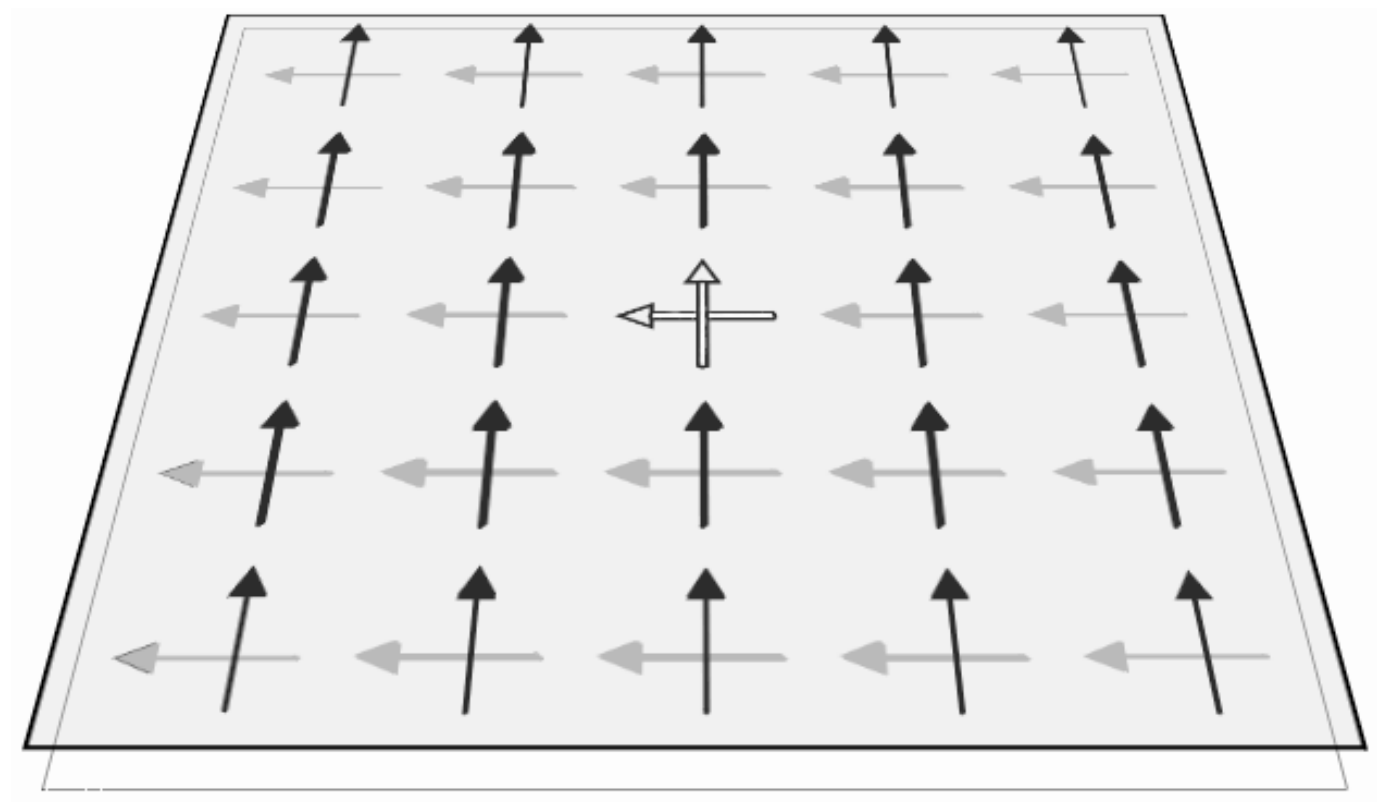

Figure 1: Structure of the array, viewed from above. Both arrays lie in the $i j$-plane, with all donor transition moments (black) in the upper array parallel to the $i$-axis, and all acceptor transition moments (gray) in the lower array parallel to the $j$-axis. The open arrows represent one excited donor and its counterpart acceptor. 
Although each donor has a geometrical counterpart acceptor, in developing the theory it is necessary to treat every acceptor as a possible target for the energy transfer. Thus it is expedient to focus on the fundamental process of energy transfer from a single excited donor, located at $r \hat{\boldsymbol{k}}$, to an arbitrarily displaced acceptor particle in the other array.

\subsection{RET between the Arrays}

The array can be constructed so that the transition moment associated with each donor particle is orthogonal to that of each acceptor; in one such way, $\boldsymbol{\mu}_{D}^{0 \alpha}$ lies along the $\hat{\boldsymbol{i}}$-axis and all $\boldsymbol{\mu}_{A}^{\beta 0}$ lie along the $\hat{\boldsymbol{j}}$-axis. The dipole transition moments and the separation vector are thus defined as;

$$
\hat{\boldsymbol{\mu}}_{A}^{0 \alpha}=\hat{\boldsymbol{i}} \quad ; \quad \hat{\boldsymbol{\mu}}_{B(u, v)}^{\beta 0}=\hat{\boldsymbol{j}} \quad ; \quad \boldsymbol{R}=u \hat{\boldsymbol{i}}+v \hat{\boldsymbol{j}}+r \hat{\boldsymbol{k}} \quad .
$$

The magnitude of the separation of the donor and an acceptor is thus;

$$
R_{(u, v)}^{6}=l^{6}\left(u^{2}+v^{2}+r^{2}\right)^{3}
$$

and the orientation factor is;

$$
\kappa(u, v)=\frac{-3(u l)(v l)}{(u l)^{2}+(v l)^{2}+r^{2}}=\frac{-3 u v}{u^{2}+v^{2}+r^{\prime 2}},
$$

with $r^{\prime}=r / l$. Substituting (9) and (10) into (2) gives the following probability for excitation transfer from the donor at an arbitrary origin $(0,0)$ to an acceptor in the other plane at $(u, v)$;

$$
P_{u v}=K t \frac{\kappa^{2}}{\left(u^{2}+v^{2}+r^{\prime 2}\right)^{3}}=\left(\frac{\left|\mu^{A}\right|^{2}\left|\mu^{B}\right|^{2} \rho_{f} t}{8 \pi \hbar \varepsilon_{0}^{2} l^{6}}\right) \frac{9 u^{2} v^{2}}{\left(u^{2}+v^{2}+r^{\prime 2}\right)^{5}} .
$$

As can be seen from (11), and as required, RET is precluded between each donor and its counterpart acceptor $(u=v=$ 0 ); in fact there will be no energy transfer to any acceptor that lies along either the $\hat{\boldsymbol{i}}$ - or $\hat{\boldsymbol{j}}$-axis, including the closest acceptor to the donor, since if either $u$ or $v$ is zero, $P_{u v}=0$. Figure 2 shows the relative probability of energy transfer to each acceptor in the array, with $r^{\prime}=0.1$.

$$
\log (P / K t)
$$
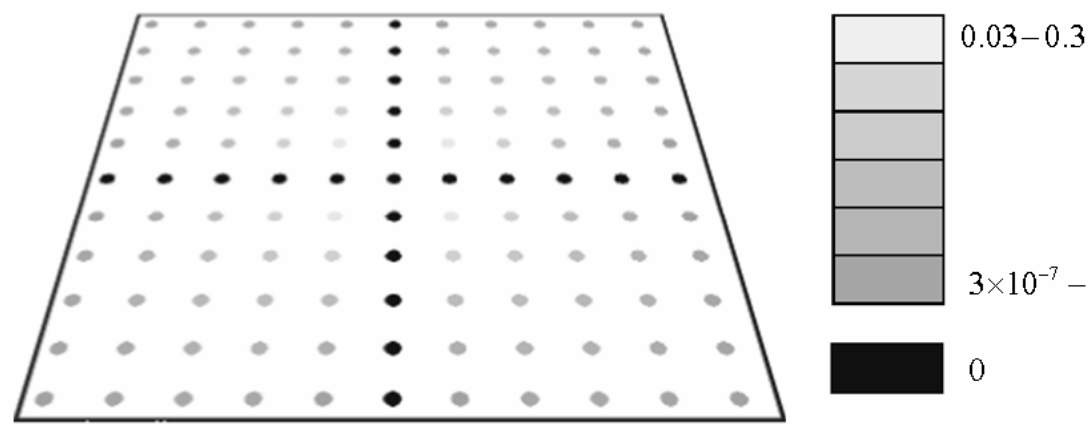

$0.03-0.3$

$3 \times 10^{-7}-3 \times 10^{-6}$

Figure 2: Energy transfer probabilities to each acceptor, from a donor located above the centre of the array, with no external influences. Each dot represents an acceptor, the brightness of which indicates the transfer probability. 


\subsection{LARET between the Arrays}

Given the arrangement described above, energy transfer from a donor to its counterpart acceptor requires an external source to activate the LARET mechanism. The $\alpha$ tensor (6) relates to a generalized dynamic polarizability, characterized by the sum over all possible electronic states $r$ of the chromophore - the largest contributors to this sum are those transitions closest in energy to the energy of photons in the forward-scattered radiation. To expedite calculations involving polarizabilities, it is common to employ a two-level approximation to the chromophores - in other words assuming that all other energy levels besides the initial and final ones are of significantly higher energy, and as such provide only minimal corrections to the result. In this instance, however, the transition between the donor and acceptor states $\alpha$ and $\beta$ and their corresponding electronic ground states designedly prohibits energy transfer, necessitating the consideration of a third energy level for each chromophore. Although each $\alpha$ tensor contains an intermediate state sum which is effected over all electronic states of the specific species involved, generally one summand is significantly larger in magnitude than the others, because its denominator is small. This feature can be exploited by selection of a frequency for the applied radiation that has a small resonance offset, such that $E_{\sigma \alpha}=\hbar c k+\Delta E_{D}$, where $\sigma$ is one specific state of $D$ (distinct from $\alpha$ and 0) with non-zero transition moments to both $\alpha$ and $0 ; \Delta E_{D}$ is a non-zero energy with significantly lower magnitude than a typical transition energy. An equivalent near-resonant state $\tau$ can be assumed for A, although $\Delta E_{D}$ and $\Delta E_{A}$ are not necessarily equal. Analysis of eqns (5) and (6), including $\sigma$ and $\tau$ in the sum, reveals that the largest contribution to the probability (that permits energy transfer) comes from eqn (5a), which produces a denominator $\Delta E_{D} \Delta E_{A}$, significantly smaller than any other term which arises.

To further expedite the calculations, the array can be constructed such that $\boldsymbol{\mu}^{\sigma \alpha}$ and $\boldsymbol{\mu}^{\beta \tau}$ are parallel, allowing the polarization vector of the incident radiation to be set parallel to these transition moments - reinforcing this pathway. Furthermore, $\boldsymbol{\mu}^{0 \sigma}$ and $\boldsymbol{\mu}^{\tau 0}$ can be set up parallel to $\hat{\boldsymbol{k}}$. The results give by equations (10) and (11) are unchanged by the application of laser light. The orientation factor arising from the LARET pathway can be expressed in terms of $u$ and $v$ as;

$$
\kappa^{\prime}(u, v)=\frac{u^{2}+v^{2}-2 r^{2}}{u^{2}+v^{2}+r^{\prime 2}}
$$

Applying the discussion above to eqns (4) and (7) allows the overall probability of energy transfer in the presence of incident radiation to be expressed as follows, in terms of the relative position of the acceptor with respect to the donor and the irradiance of the laser light;

$$
P_{u v}(t)=K \times \frac{9 u^{2} v^{2}-6 C u v\left(u^{2}+v^{2}-2 r^{\prime 2}\right) \int_{0}^{t} I(t) d t+C^{2}\left(u^{2}+v^{2}-2 r^{\prime 2}\right)^{2} \int_{0}^{t} I^{2}(t) d t}{\left(u^{2}+v^{2}+r^{\prime 2}\right)^{5}},
$$

with $K$ as defined by equation (11); the parameter $C$ is given by;

$$
C=\frac{|\mu|^{2}}{2 c \varepsilon_{0} \Delta E_{A} \Delta E_{B}}
$$

and $I(t)$ is the time-dependent irradiance of pulsed throughput radiation. The time integrations in the numerator of (13) signify an accumulation of probability through the optical pulse. Comparing (13) to (11), it is apparent that energy can transfer with varying probabilities to all acceptors in the lower array. Specifically, the applied laser light 'switches on' energy transfer to those acceptors lying along the $\hat{\boldsymbol{i}}$ and $\hat{\boldsymbol{j}}$ axes, for which $u=0$ or $v=0$. 


\section{RESULTS AND DISCUSSION}

To achieve maximum efficiency from the array structure, the application of laser radiation should produce a large increase in the probability of transfer to the counterpart acceptor, without significantly increasing that of transfer to any other acceptor. The array configuration then represents a set of optical transistors with parallel processing capacity. Three controlling factors are apparent, one concerning the construction of the system, the other two the characteristics of the laser beam. The first of these factors to consider is the aspect ratio $r^{\prime}$. The smaller the separation of each donor from its counterpart acceptor - when compared to the displacement of any other particle - the more efficient the system will be. However, there is a minimum donor-acceptor separation of about $0.4 \mathrm{~nm}$, below which the wavefunctions of the chromophores will begin to overlap, promoting a competing Dexter exchange mechanism. Alternatively, the aspect ratio can be decreased by increasing the lattice constant of the arrays, although a larger lattice constant will signify a decrease in transistor density, defeating the objective of producing nanoscale photonics components. A suitable compromise might be an aspect ratio of 0.025 , signifying, for example, an array separation of $10 \mathrm{~nm}$ and a lattice constant of $400 \mathrm{~nm}$. The second factor is the frequency of the laser pulse. Tuning the laser frequency close to resonance will in principle increase LARET efficiency, but it also introduces the risk of promoting other mechanisms (single-photon absorption and/or stimulated emission). Finally, the irradiance of the laser beam needs also to be considered. The term of interest in eqn (13) has a quadratic dependence on the irradiance, so the higher the irradiance, the higher the probability of energy transfer. If irradiated with a beam of intensity $10^{16} \mathrm{~W} \mathrm{~m}^{-2}, r^{\prime}=0.1$ and with an optical frequency such that $\Delta E=3.3 \times 10^{-20} \mathrm{~J}$ (about one-tenth of a typical molecular transition), energy transfer between counterpart donors and acceptors will be approximately $10^{5}$ more effective than to between any other pair of chromophores. Conversely, an aspect ratio of $1: 40\left(r^{\prime}=0.025\right)$ permits a more modest irradiance of $10^{14} \mathrm{~W} \mathrm{~m}{ }^{-2}$ to achieve a similar level of effectiveness, as in figure 3 . As this shows, the application of laser radiation vastly increases the transfer probability to the intended acceptor without affecting transfer to any other acceptor.

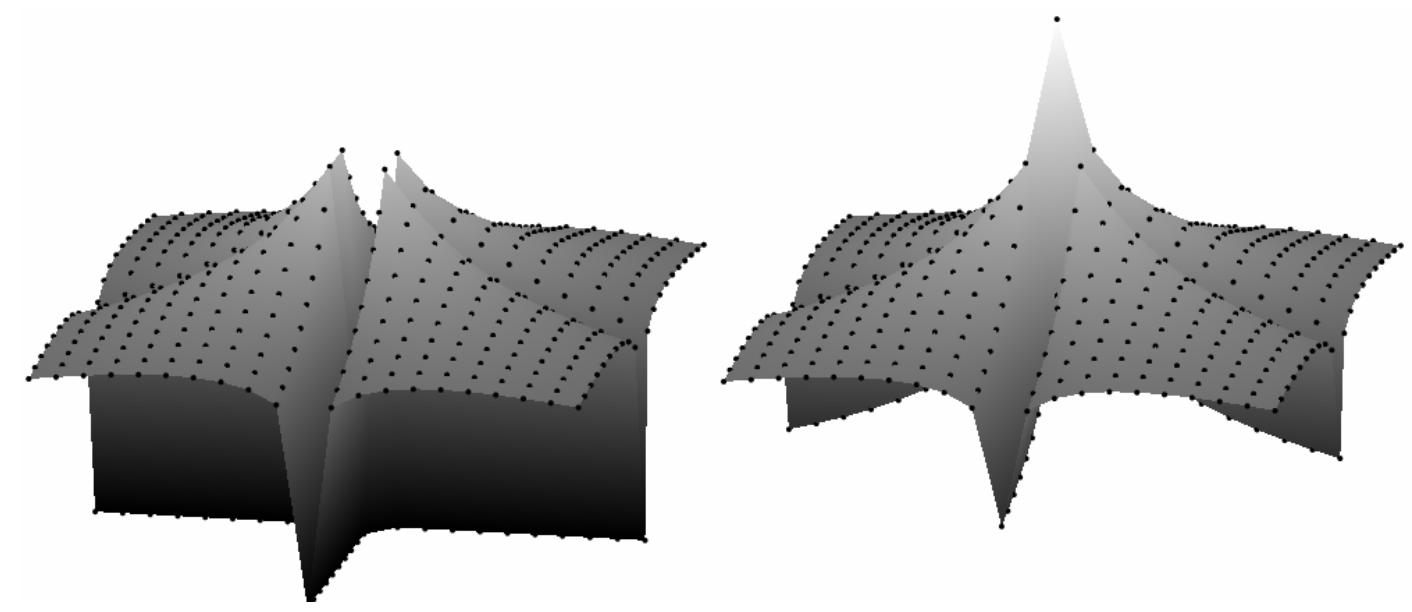

Figure 3: Contour graph depicting the probability of energy transfer to the array of acceptors in the absence (left) and presence (right) of laser light. The probability of energy transfer is represented by the vertical scale (plotted on a logarithmic scale), and the position of each acceptor has been shown for clarity.

To minimize other competing processes which might represent losses of information or fidelity, a principle widely used in multichromophore systems for conventional RET could be introduced. Specifically, back-transfer from acceptors to donors can be precluded by engineering differences in the absorption and emission profiles of the particles. This means of ensuring directionality is widely known as a spectroscopic gradient or energy funnel. ${ }^{11}$ The sought advantage of unidirectionality which is thereby ensured is offset by a reduction in efficiency necessarily associated with small losses of energy through vibrational relaxation or phonon generation, possibly necessitating the adoption of appropriate cooling measures in device applications. The other potential complication, the intrinsically finite probability for energy transfer from the designated acceptor to another acceptor, can be addressed by configuration of the relative 
the relative spacing of the donor and acceptor arrays, relative to the internal spacing of the particles in each array. It is submitted that the results promise a new basis for the design of optically activated transistor action in nanoscale components.

\section{ACKNOWLEDGEMENTS}

This research from the QED group at the University of East Anglia is funded by the UK Engineering and Physical Sciences Research Council.

\section{REFERENCES}

1. A. Aviram, "Molecules for Memory, Logic, and Amplification", J. Am. Chem. Soc., 110, pp. 5687-5692, 1988.

2. T. Rueckes, K. Kim, E. Joselevich, G. Y. Tseng, C. L. Cheung and C. M. Lieber, "Carbon nanotube-based nonvolatile random access memory for molecular computing", Science, 289, pp. 94-97, 2000.

3. B. S. Ham, "A novel method of all-optical switching: Quantum router", Etri J., 23, pp. 106-110, 2001.

4. $\quad$ D. Geppert, L. Seyfarth and R. de Vivie-Riedle, "Laser control schemes for molecular switches", Appl. Phys. B-Lasers O., 79, pp. 987-992, 2004.

5. O. Wada, "Femtosecond all-optical devices for ultrafast communication and signal processing", New J. Phys., 6, pp. 183, 2004.

6. P. Allcock, R. D. Jenkins and D. L. Andrews, "Laser-assisted resonance-energy transfer", Phys. Rev. A, 6102, pp. 023812, 2000.

7. D. P. Craig and T. Thirunamachandran, Molecular Quantum Electrodynamics: An Introduction to RadiationMolecule Interactions (Academic Press, London, 1984).

8. G. J. Daniels, R. D. Jenkins, D. S. Bradshaw and D. L. Andrews, "Resonance energy transfer: The unified theory revisited", J. Chem. Phys., 119, pp. 2264-2274, 2003.

9. G. Juzeliunas and D. L. Andrews, "Unified theory of radiative and radiationless energy transfer" in Resonance Energy Transfer (Wiley, Chichester, 1999).

10. D. L. Andrews and R. G. Crisp, "Optically-Activated Energy Transfer: Array Implementation", J. Opt. A: Pure Appl. Opt., (in press).

11. D. L. Andrews and R. G. Crisp, "Theory of Directed Electronic Energy Transfer", J. Fluor., (in press). 